\title{
Der Erwerbseinstieg junger Erwachsener: unsicher, ungleich, ungesund
}

\section{Thomas Langhoff Ina Krietsch Christian Starke}

\begin{abstract}
Die vielfältigen Erwerbseinstiege junger Erwachsener sind von Unsicherheiten geprägt. Vor allem diese Gruppe ist an den verschiedenen Übergangsstellen - vom Schul- ins Ausbildungssystem und anschließend ins Berufsleben - zunehmend mit zahlreichen Schwierigkeiten und Hindernissen konfrontiert. Diese führen auch dazu, dass die Förderung und der Erhalt der psychischen Gesundheit der heutigen jungen Generation aufgrund ihrer Arbeitsmarkt- und Beschäftigungsbedingungen stark beeinträchtigt werden. Die heterogenen Problemlagen dieser sowohl schwer greifbaren als auch schwer zugänglichen Gruppe von Beschäftigten bzw. Arbeits- und Ausbildungsplatzsuchenden sprechen wir hier als Kohorten- bzw. Generationeneffekt an.
\end{abstract}

\section{Einleitung}

Bei den Gewerkschaften wächst die Sensibilität für die besonderen Probleme der jungen Generation auf dem deutschen Arbeitsmarkt, wie verschiedene Veranstaltungen und Debatten der letzten Monate zeigen. Thematisiert werden die heterogenen Problemlagen dieser Gruppe, die zunehmend an den Übergängen vom Schul-ins Ausbildungs- und anschließend ins Berufsleben mit zahlreichen Schwierigkeiten und Hindernissen konfrontiert ist (vgl. HBS/DGB 2009). Der Beitrag greift diesen Gedanken auf, beschreibt die empirisch beobachtbare, vielfältige Praxis des Einstiegs junger Erwachsener in das Arbeitsleben und charakterisiert bestimmte Schwellen für spezifische Gruppen. Dabei geht es auch um die Frage, wie diese Schwierigkeiten auf die psychische Gesundheit der jungen Erwachsenen wirken. Unsere These ist, dass die Förderung und der Erhalt der psychischen Gesundheit dieser Generation aufgrund ihrer Beschäftigungsbedingungen gegenwärtig stark vernachlässigt werden.

In der Presse begegnen dem Leser häufig Schlagzeilen wie „Generation Praktikum“ oder "Generation prekär“. Damit wird für ein bestimmtes Arbeitsmarktsegment - auf die schwierige Situation Jugendlicher und junger Erwachsener aufmerksam gemacht, die sich auf der Schwelle vom Ausbildungs- ins Berufsleben befinden. Doch Fuß zu fassen auf dem Arbeitsmarkt, stellt nicht nur für viele Jugendliche und junge Erwachsene der mittleren und höheren
Ausbildungsgänge, die auf der Suche nach geeigneten Praktikumsplätzen sind, eine große Herausforderung dar. Auch andere Qualifikationsgruppen sind mit einem ernsthaften Problem konfrontiert. Die schwierige Suche nach geeigneten betrieblichen Ausbildungsplätzen kennzeichnet den Arbeitsmarkt für Schulabgänger aus den Haupt- und Realschulen bereits seit langer Zeit. Inzwischen - darauf weist auch die seit einigen Jahren anhaltende Prekaritätsdebatte hin - betreffen diese problematischen Arbeits- und Ausbildungsmarktzugänge breitere Bevölkerungsschichten und insbesondere einen Großteil der jungen Generation.

Aktuell treffen die Auswirkungen der Finanzkrise junge Menschen besonders hart: Die Arbeitslosigkeit ist in der Altersgruppe der unter 25-Jährigen mit $17 \%$ seit Beginn der Wirtschaftskrise beinahe dreimal so stark gestiegen wie bei allen anderen Altersgruppen (BA 2009). Es wundert also nicht, dass die Angst vor Arbeitslosigkeit oder Arbeitsplatzverlust bereits von Schülern geäußert wird (Stangl-Taller 2009; Deutsche Shell 2002). Damit werden Jugendliche $\mathrm{zu}$ einer der benachteiligten Gruppen, die drohende Arbeitslosigkeit, also hohe Zukunftsunsicherheit, bereits als eine prägende Generationenerfahrung erleben.

In Bezug auf den schwierigen Berufseinstieg Jugendlicher und junger Erwachsener ist nicht nur die Zunahme der Arbeitslosigkeit als zentraler Trend identifizierbar (Abschnitt 2). Insbesondere das Absolvieren von Praktika (Abschnitt 3) und prekäre Beschäftigung (Abschnitt 4) stellen für die Betroffenen häufig die einzige Möglichkeit dar, der schwierigen Suche nach einem geeigneten Ausbildungsplatz (Abschnitt 5 und 6) oder der drohenden Arbeitslosigkeit zu begegnen.

Im vorliegenden Beitrag werden ganz bewusst Trends diskutiert, die verschiedene Qualifikationsgruppen und Arbeitsmarktsegmente betreffen, um die Problematiken des Einstiegs junger Erwachsener in der Breite zu veranschaulichen. Diese Trends führen gleichsam zu einer Zunahme erlebter Unsicherheit und Ungleichheit unter teilweise objektiv prekären Arbeitsbedingungen, die bei den jungen Erwachsenen pathogene Entwicklungen in Gang setzen, deren Auswirkungen für die Zukunft besorgniserregend sind. Schließlich wird das Augenmerk auf die besondere Häufung von Risiken für die psychische Gesundheit, denen junge Beschäftigte

\footnotetext{
Thomas Langhoff, Prof. Dr., Hochschule Niederrhein, ist Geschäftsführer der Prospektiv GmbH Dortmund. Arbeitsschwerpunkte: Demografischer Wandel, angewandte Arbeitsforschung, psychische Gesundheit.

e-mail: langhoff@prospektiv-do.de Ina Krietsch, wissenschaftliche Mitarbeiterin bei der Prospektiv GmbH Dortmund. Arbeitsschwerpunkte: Arbeits- und Gesundheitsschutz, neue Beschäftigungsverhältnisse und Arbeitsformen, Personal- und Organisationsentwicklung. e-mail: krietsch@prospektiv-do.de Christian Starke, studentischer Mitarbeiter bei der Prospektiv GmbH Dortmund, Arbeitsschwerpunkt: Interkulturelle Kompetenz, Leiharbeit. e-mail: info@prospektiv-do.de
} 
verstärkt ausgesetzt sind, gerichtet (Abschnitt 7). Im Fazit (Abschnitt 8) wird vor allem auf bereits vorhandene Konzepte und Strategien hingewiesen, mit denen diese spezifische Ungleichbehandlung der jungen Generation zu entschärfen wäre.

\section{Jugendarbeitslosigkeit}

Eine im März 2009 im Auftrag der IG Metall durchgeführte Studie zeigt, dass junge Arbeitnehmerinnen und Arbeitnehmer sechsmal häufiger ihren Arbeitsplatz verlieren als Erwerbspersonen, die älter als 35 Jahre sind. Vier von zehn jungen Erwerbstätigen ( $37 \%$ ) haben ihren Arbeitsplatz bislang schon ungewollt wechseln müssen; $32 \%$ der jungen Arbeitnehmerinnen und Arbeitnehmer waren bereits mehr als sechs Monate arbeitslos. Dennoch wird die Jugendarbeitslosigkeit häufig unterschätzt. Das mag unter anderem daran liegen, dass Jugendliche, die eine Ausbildungsstelle suchen und parallel dazu nicht alternativ auf der Suche nach einer Arbeitsstelle sind, von der Arbeitslosenstatistik nicht erfasst werden. Auch führen berufsfördernde Maßnahmen, in die arbeitslose Jugendliche vermittelt werden (z. B. Weiterbildungsmaßnahmen oder Ein-Euro-Jobs), zu einer Grauzone bei der Erfassung der Jugendarbeitslosigkeit. Auf der anderen Seite ist festzustellen, dass der Arbeitsmarkt für Jüngere schnelllebiger als für ältere Arbeitnehmer ist. Das bedeutet, dass Jugendliche zwar von einem doppelt so hohen Risiko, arbeitslos zu werden, betroffen sind, allerdings ist die Dauer der Arbeitslosigkeit bei jungen Erwachsenen um ein Vielfaches kürzer als bei älteren Erwerbspersonen. Trotzdem stellt Arbeitslosigkeit für Jugendliche und junge Erwachsene die zentrale Hürde beim dauerhaften Einstieg ins Berufsleben dar.

Die Folgen von Arbeitslosigkeit, Arbeitsplatzunsicherheit und Misserfolg bei der Ausbildungsplatzsuche sind für die Betroffenen weitreichend: Erwerbsarbeit nimmt auf der persönlichen wie gesellschaftlichen Ebene einen sehr hohen Stellenwert ein und bedeutet für die Betreffenden mehr als die Sicherstellung des täglichen Lebens. (Erwerbs-)Arbeit bedeutet idealtypisch Teilhabe an der Gesellschaft, Zugang zu sozialen Netzwerken, Beitrag zu einem großen Ganzen, Entfal- tung von Persönlichkeit und Selbstverwirklichung. Der Verlust des Arbeitsplatzes bzw. die Nicht-Zugänglichkeit des Arbeitsmarktes hat für die betroffenen Personen daher weitreichende Konsequenzen - über die rein finanzielle Notlage hinaus. Für Jugendliche und junge Erwachsene bedeutet Arbeitslosigkeit die Gefahr von Entwicklungsbeeinträchtigungen (Stangl-Taller 2009). Die Erfahrung, nicht gebraucht zu werden, kann u.a. zu vermindertem Selbstvertrauen, zum Verlust sozialer Kontakte, zur psychischen Destabilisierung und sogar zu Depressionen führen. Der Verlust des Arbeitsplatzes gehört generell zu den traumatisierendsten Lebenserfahrungen. Bereits ab der Jugendphase solchen psychischen Belastungen ausgesetzt zu sein, erhöht die Wahrscheinlichkeit, im späteren Berufsleben an körperlichen und vor allem psychischen Erkrankungen zu leiden (Rump/Eilers 2009).

Prager/Wieland (2005) fanden im Rahmen einer Umfrage der Bertelsmann-Stiftung heraus, dass mehr als jeder zweite Befragte die persönlichen Ausbildungs- und Berufschancen mit Skepsis und Pessimismus betrachtet. Der Hauptgrund für diese Einschätzung liegt nach Angaben der Befragten an der mangelnden Verfügbarkeit von Arbeitsplätzen (81 \%). Fast zwei Drittel (63\%) der befragten Jugendlichen sind allerdings auch bereit, „wirklich“ etwas für ihren beruflichen Erfolg zu tun und „Opfer zu bringen"-zum Beispiel das Absolvieren eines unbezahlten Praktikums.

\section{Praktikum - Ausbeutung
oder Chance?}

Viele Jugendliche und junge Erwachsene versuchen, über den Weg eines Praktikums den Einstieg ins Erwerbsleben zu schaffen. Diese Beschäftigungsform kann als „prekär ${ }^{\text {“1 }}$ bezeichnet werden, wenn Praktikanten keine Vergütung erhalten, entweder zu verantwortungsvolle oder aber unterqualifizierte Tätigkeiten übernehmen oder täglich länger als zwölfStunden sowie abends und an Wochenenden arbeiten. Genau diese Merkmale kennzeichnen jedoch einen Großteil der Praktikumsplätze. Auch erhalten einige keinen Praktikumsvertrag, der Dauer, Inhalte und Vergütung des Praktikums regelt etc. Junge Erwachsene nehmen diese prekären Bedingungen hin, weil sie Standards (Arbeitsverträge, Arbeitszeiten u.ä.) nicht kennen und ein Praktikum der Arbeitslosigkeit vorziehen.

Im Jahr 2007 haben insgesamt knapp 600.000 Berufsanfänger ein Praktikum absolviert (IAB 2007). Dabei stellt sich häufig die Frage, ob diese Beschäftigungsform auf eine kostengünstige Aneignung von Arbeitskraft und Arbeitsleistung zielt, also auf Ausbeutung, oder ob sie eine ernstzunehmende Chance zum Eintritt in den Arbeitsmarkt darstellt. Tatsächlich verbindet sich damit die Möglichkeit, Erfahrungen zu sammeln, Wissen zu generieren und Einblicke in die Berufswelt zu bekommen. Absolventen erhoffen sich über ein Praktikum häufig nicht nur eine Möglichkeit der Qualifizierung, sondern auch eine Anschlussbeschäftigung oder den Aufbau eines sozialen Netzes (Mörchen 2006). Auf der anderen Seite enden jedoch zahlreiche Praktika ohne den erhofften Arbeitsvertrag.

Das Bundesarbeitsgericht (BAG) definierte 2003 einige verbindliche Standards für Praktikumsplätze: „Praktikant ist, wer sich für eine vorübergehende Dauer zwecks Erwerb praktischer Kenntnisse und Erfahrungen einer bestimmten betrieblichen Tätigkeit und Ausbildung, die keine systematische Berufsausbildung darstellt, im Rahmen einer Gesamtausbildung unterzieht, weil er diese für die Zulassung zum Studium oder Beruf, zu einer Prüfung oder anderen Zwecken benötigt" (BAG 13.03.03 - 6 AZR 564/01).

Im Gegensatz zu der BAG-Entscheidung gehen Praktikanten jedoch häufig unterqualifizierten Tätigkeiten nach und/ oder sie ersetzen sogar reguläre Beschäftigung. Praktika werden von Unternehmen durchaus als einfaches und kostengünstiges Rekrutierungsinstrument genutzt. Inzwischen ist auf diese Weise das Segment „Praktikantenarbeitsmarkt“ mit eigenen Anspracheformen (Messen, Veranstaltungen), Rekrutierungswegen (Internet-Plattformen) samt Bewerbungsstrategien und zugehöriger Ratgeberliteratur entstanden. Diese Entwicklung vollzieht sich nicht nur in Deutschland. In Frankreich beispielsweise wurde im Jahr 2006 von noch höhe-

\footnotetext{
1 Der Begriff bedeutet unsicher, schwierig oder problematisch und macht auf die unsicheren und zum Teil sehr schlechten Arbeitsbedingungen bestimmter atypischer Beschäftigungsverhältnisse aufmerksam (Niedriglohn, geringer Kündigungsschutz, wenig soziale Sicherung, Statusprobleme etc.).
} 
ren Fallzahlen bzgl. Praktika mit ähnlich prekären Verhältnissen berichtet (Wesfreid 2006).

Die sozialen und ökonomischen Konsequenzen sind nicht nur für die Betroffenen, sondern auch für die Volkswirtschaft enorm: Aufgrund des geringen oder nicht vorhandenen Gehalts zahlen Praktikanten keine Steuern und keine Beiträge in die sozialen Sicherungssysteme. Für die Praktikanten ist die schwierige Einkommenssituation häufig gleichbedeutend mit Armut und sozialen Problemen. Sie führen so zu einem pessimistischen Blick auf die eigene Zukunft. Junge Erwachsene mit prekären Arbeitsverhältnissen sind nicht in der Lage, ihre Zukunft zu planen (z. B. Gründung einer Familie, Anschaffungen, Weiterqualifizierung), was die demografischen Probleme wiederum verschärft. Abgesehen von der Situation der betroffenen Praktikanten kommt es auch zu Verzerrungen im Wettbewerb, wenn Unternehmen außergewöhnlich viele Praktikanten beschäftigen, um Lohnkosten zu sparen (Generation P 2007).

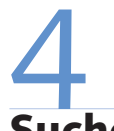

\section{Suche nach Ausbildungsplätzen}

Glücklich kann sich schätzen, wer direkt, ohne lange Wartezeiten und eingeschobene Praktika einen Ausbildungsplatz findet. Doch die Suche wird für Jugendliche und junge Erwachsene häufig bereits zu einem Problem. 2008 bildeten weniger als $25 \%$ der Betriebe in Deutschland aus. Dabei scheint die Regel zu gelten: Je größer der Betrieb, desto geringer die Quote an Auszubildenden.

Den sinkenden Zahlen abgeschlossener Ausbildungsverträge und Stellenangebote steht derzeit ein Rückgang von Schulabgängern gegenüber - die Vorboten des demografischen Wandels. Trotzdem sind immer noch Tausende auf der Suche nach einer Ausbildungsstelle, Altbewerber haben es dabei besonders schwer (BMBF 2009).

Auch der Berufsbildungsbericht des Bundesministeriums für Bildung und Forschung weist auf die Ambivalenz der Situation auf dem Ausbildungsmarkt hin (BMBF 2009). Auf der einen Seite entspanne sich zwar seit 2006 das Verhältnis von Angebot und Nachfrage an Ausbil- dungsplätzen. Dennoch blieben im Jahr 2008 über 9.600 Bewerber ohne Ausbildungsvertrag. Im Vergleich zum Vorjahr beträgt der Rückgang an abgeschlossenen Ausbildungsverträgen 1,5\%. Der Bericht macht deutlich, dass die Auswirkungen der „Finanzkrise“ nur schwer einzuschätzen sind, da der Ausbildungsmarkt eng mit der Wirtschaftslage verbunden ist.

Der schwierige Weg für Jugendliche vom Schulabschluss bis zur Unterzeichnung eines Ausbildungs- bzw. Arbeitsvertrages lässt sich ungeachtet der qualifikationsspezifischen Unterschiede als Labyrinth der Ausbildungs- bzw. Arbeitsplatzsuche charakterisieren. Es hält zwar viele Wege bereit, jedoch auch einige Sackgassen, wie die der Jugendarbeitslosigkeit, oder die Umwege über Praktika und atypische Beschäftigung hin zum Ausbildungs- bzw. Arbeitsvertrag.

Doch auch für diejenigen Schulabgänger, die am Ziel des Irrwegs angekommen einen Ausbildungsplatz gefunden haben, ist im Anschluss an die Ausbildung die Übernahme seitens des Ausbildungsbetriebs und somit der Arbeitsplatz nicht sicher. Im Jahr 2007 wurden $40 \%$ der Auszubildenden nicht von den Ausbildungsbetrieben übernommen (Fischer et al. 2007). Auch hier ist noch offen, inwiefern die Finanz- und Wirtschaftskrise die Situation verschärfen wird, wenn sie auf den Arbeitsmarkt durchschlägt, spätestens nach Wegfall der Kurzarbeiterregelung. Mit Zunahme der Arbeit Suchenden werden sich sicherlich im Labyrinth - um im Bild zu bleiben - mehr Menschen verirren, in Sackgassen landen, Umwege gehen müssen, um dann doch nicht ans Ziel zu kommen - so ist zu befürchten.

Selbst bei denen, die die erste Schwelle von der Schule in die berufliche Ausbildung geschafft haben, stellt sich nach kurzer Zeit wieder die Frage, wie es weitergeht: Was bei Nicht-Übernahme folgt, ist, wie die Statistik zeigt, häufig die Arbeitslosigkeit oder der Weg in atypische, prekäre Beschäftigung, die im Folgenden genauer beleuchtet wird.

\section{Atypische Beschäftigung}

Der ehemals klassische berufliche Werdegang, der die Erwerbsperson von der Ausbildung oder einem Studium direkt in den Betrieb führt, wo sie einer unbefristeten Vollzeitstelle nachgeht, die idealerweise bis zur Erreichung des Rentenalters ${ }^{2}$ beibehalten wird, ist heute in dieser Form kaum noch vorzufinden. Dem beschriebenen „Normalarbeitsverhältnis“ stehen gegenwärtig zahlreiche atypische Beschäftigungsverhältnisse gegenüber. Damit sind Anstellungsverhältnisse wie Teilzeit, Heimarbeit, Mini- und Midijobs sowie Leiharbeit gemeint. Atypische Beschäftigungsverhältnisse gehen häufig einher mit prekären Arbeitsbedingungen (Niedriglohn, geringer Kündigungsschutz, Statusprobleme, geringe soziale Sicherung etc.). Ob befristet Beschäftigte oder Erwerbspersonen in Teilzeit ihre Arbeitsverhältnisse subjektiv auch als „prekär“ bezeichnen bzw. wahrnehmen, muss an dieser Stelle nicht erörtert werden. Dass jedoch Beschäftigung in Leiharbeit und in geringfügigen Arbeitsverhältnissen schwierige Bedingungen für die Betreffenden bedeuten, dürfte unstrittig sein, wie verschiedene Untersuchungen, die auch nach der subjektiven Bewertung der Beschäftigungssituation fragen, zeigen.

Jüngst berichtete das statistische Bundesamt von einer massiven Zunahme befristeter Beschäftigung als Krisenbewältigungsstrategie der Unternehmen. Vor allem für größere Unternehmen (laut IAB $90 \%$ der Betriebe ab 250 Beschäftigten) ist Befristung bei Einstellung gängige Praxis. Die Süddeutsche Zeitung titelte deshalb sogar: „Unsicherheit wird zur Regel“ (17.3.2010). Der Trend zur Prekarisierung bleibt mit dieser - meist von den Beschäftigten ungewollten - Arbeitsvertragsgestaltung weiterhin ungebrochen.

Abbildung 1 zeigt die Zunahme des Anteils atypisch Beschäftigter in den Jahren zwischen 1997 und 2007. Auffallend ist jedoch nicht nur der generelle Anstieg atypischer Beschäftigungsverhältnisse, sondern darüber hinaus der signifikant wachsende Anteil der Unter-25-Jährigen an dieser Beschäftigungsform. In dieser Gruppe hat sich der Anteil atypisch Beschäftigter an den abhängig Beschäftigten innerhalb von zehn Jahren verdoppelt. Inzwischen sind $39 \%$ aller Beschäftigungsverhältnisse bei den Unter-25-Jährigen atypischer Art, bei der Gruppe zwischen 15 und unter 20 Jah-

2 Wir sehen hier von Erwerbsunterbrechungen aufgrund familiärer Verpflichtungen $a b$, nach denen man bisher weitgehend mit einer Rückkehr in den Betrieb rechnen konnte. 
ren weist die Statistik für 2010 sogar $41 \%$ aus (Grau 2010, S. 2).

Befristete Beschäftigung kann auch als Einstieg in die Erwerbsarbeit gelten. $\mathrm{Ob}$ der Einstieg allerdings in ein stabiles Normalarbeitsverhältnis übergeht, ist fraglich. Fuchs (2008) berichtet aus einer BAuA/ BMAS-Untersuchung, dass lediglich $23 \%$ der qualifizierten Jugendlichen nach ihrem befristeten Arbeitsverhältnis (Übernahme nach der Ausbildung) in ein stabiles Beschäftigungsverhältnis gekommen sind. Auch das Statistische Bundesamt hat gezeigt, dass Befristung nicht nur ein Einstieg in ein stabiles Beschäftigungsverhältnis sein, sondern auch den Einstieg in unstete Beschäftigungskarrieren bedeuten kann, vor allem bei fehlendem Berufsabschluss (zitiert nach Kock 2008).

Unter arbeitswissenschaftlichem Blickwinkel erhalten wir Hinweise auf besondere Muster psychischer Belastungen bei atypisch Beschäftigten. Eine britische Langzeitstudie wies beispielsweise nach, dass insbesondere eine spezifische Form befristeter Beschäftigung, nämlich Saisontätigkeiten und Gelegenheitsjobs, signifikant negativen Einfluss auf die psychische Gesundheit und die Job-Zufriedenheit haben (Bardasi/Francesconi 2004). Die höhere Unsicherheit hinsichtlich der Entlohnungsbedingungen und Arbeitsmarkt-/ Personalentwicklungschancen (z.B. in Form einer Übernahme aus befristeter Beschäftigung) erhöht - wie im Gratifikationskrisenmodell beschrieben (Siegrist/ Dragano 2008) - das Risiko für stressassoziierte Krankheiten erheblich. Vor dem Hintergrund der beschriebenen Altersstrukturen dieser Beschäftigungsformen müssen wir annehmen, dass es gerade die Jüngeren sind, die den beschriebenen gesundheitlichen Risiken ausgesetzt sind.

\section{Leiharbeit - eine zuge- spitzte Form prekärer Beschäftigung}

Als exemplarisch für atypische Beschäftigungsverhältnisse - im Sinne der Häufung von psychischen Belastungen und Erkrankungsrisiken - kann die Leiharbeit angesehen werden, die im Folgenden genauer vorgestellt wird. Insbesondere bei der Leiharbeit fällt auf, dass vor allem junge Menschen dieser Beschäftigung nachgehen.

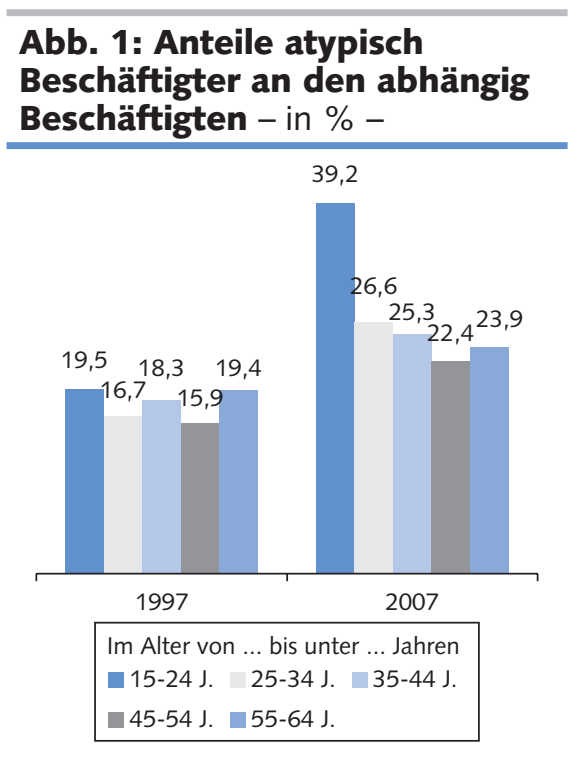

Quelle: Darstellung der Autoren.

WSI MITTELLUNGeN

Vergleicht man die Alterszusammensetzung der atypischen Beschäftigungsform Leiharbeit mit der Alterszusammensetzung der Erwerbsarbeit insgesamt, also Stammarbeits- und Leiharbeitsverhältnisse zusammen (Abbildung 2), zeigt sich, dass auch hier die Altersstruktur insbesondere durch einen überdurchschnittlich hohen Anteil junger Arbeitnehmer geprägt ist.

Umgekehrt gilt, je älter die Erwerbspersonen, desto unwahrscheinlicher wird Leiharbeit als prägende Beschäftigungsform. Insgesamt sind über $50 \%$ der Leiharbeitskräfte unter 35 Jahre. Das ist ein vergleichsweise hoher Anteil: So sind im verarbeitenden Gewerbe insgesamt weniger als $30 \%$ der Beschäftigten jünger als 35 Jahre (Sczesny et al. 2008). Diese jungen Leiharbeitskräfte werden dem Arbeitsmarkt demnach noch weitere 30-35 Jahre zur Verfügung stehen (müssen).

Im Rahmen betrieblicher Bestandsaufnahmen des Projektes GRAziL ${ }^{3}$ sowie Befragungen der IG Metall NRW wurden im subjektiven Urteil geäußerte Belastungen von insgesamt 790 Leiharbeitskräften zusammengetragen. Die Befragungen sind zum Teil standardisiert durchgeführt worden, aber auch Ergebnisse von Gruppenbefragungen und Workshops wurden verwertet. Die Befragungen in GRAziL sind in vier Produktionsbetrieben (zusammen 157 Leiharbeitskräfte) sowie in vier Dienstleistungsbetrieben (zusammen 251 Leiharbeitskräfte) durchgeführt worden. Ergänzt wurden die Daten aus GRAziL um Daten der IG Metall NRW von insge-

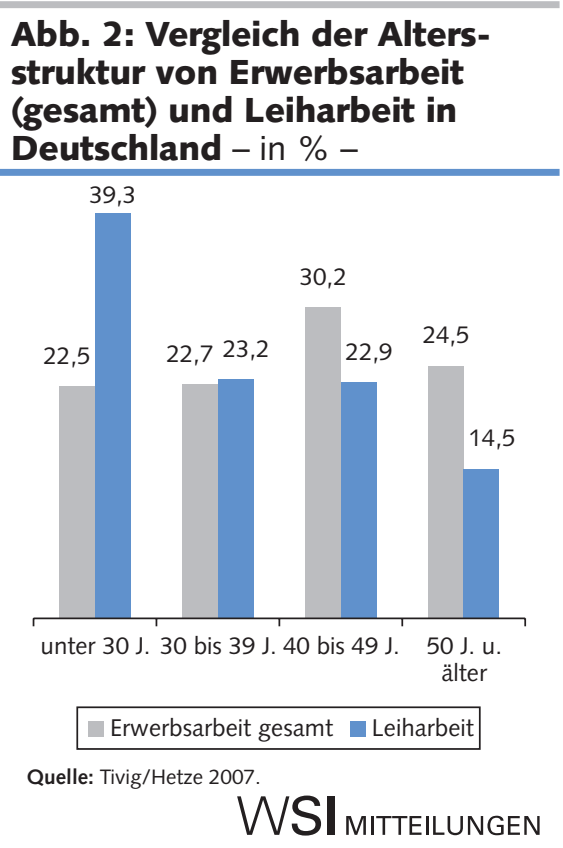

samt 382 Leiharbeitskräften aus weiteren Metallbetrieben. Die auf diese Weise gesammelten leiharbeitstypischen Belastungen wurden gelistet und inhaltlich zu acht Belastungsdimensionen geclustert (Abbildung 3). Um Ursache-Wirkungsbeziehungen dieser Belastungscluster untereinander systematisch zu betrachten, nahmen elf Arbeitswissenschaftler im Rahmen eines Expertenworkshops zu psychischen Belastungen in der Leiharbeit eine Ursache-Wirkungs-Analyse mit dem Instrument der Paarvergleichsmethode (Vester 2001) vor.

Die Analyse ergab, dass der Cluster „Problematische Verfahrensweisen des Zeitarbeitsunternehmens" (z. B. inkorrekte Lohnabrechnung, mangelhafte Betreuung etc.) die mit Abstand stärkste Wirkung auf die anderen Belastungscluster ausübt und somit als Treiber im Ursache-Wirkungsgeflecht fungiert. Die Analyse ergab außerdem, dass Leiharbeitskräfte sich aufgrund ihres Beschäftigungsverhältnisses strukturell in den Entleihunternehmen ausgegrenzt fühlen. Damit sind z.B. das

3 Das Projekt GRAziL - Gestaltung, Umsetzung und Transfer von Instrumenten zum Ressourcenmanagement und zum Arbeitsschutz im Rahmen eines zielgruppenbezogenen Ansatzes für Leiharbeitnehmer in Entleihunternehmen wird im Rahmen des Modellprogramms zur Bekämpfung arbeitsbedingter Erkrankungen vom Bundesministerium für Arbeit und Soziales (BMAS) gefördert, durch die Bundesanstalt für Arbeitsschutz und Arbeitsmedizin (BAuA) fachlich und durch die Gesellschaft für soziale Unternehmensberatung $\mathrm{mbH}$ (gsub) administrativ begleitet. 


\section{Abb. 3: Ursache-Wirkungs-Kette psychischer Belastungen} in der Leiharbeit

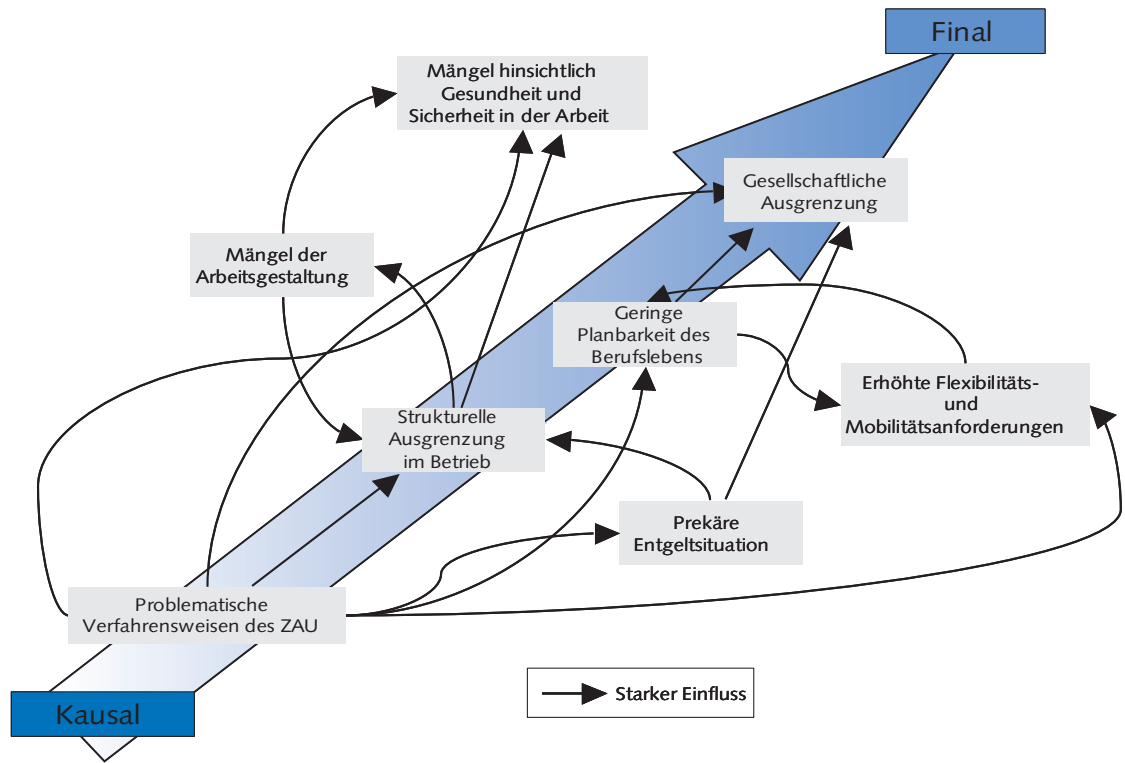

Anmerkungen: Zentrale Belastungscluster: „Problematische Verfahrensweise des ZAU“ (z.B. inkorrekte Lohnabrechnungen), "strukturelle Ausgrenzung im Betrieb" (z.B. gesonderte Arbeitskleidung), „Prekäre Entgeltsituation“ (z.B. ungleicher Lohn bei gleicher Arbeit; ZAU = Zeitarbeitsunternehmen. Quelle: Darstellung der Autoren.
Beeinträchtigung stark erhöht. $40 \%$ der Betroffenen zeigen chronische Verläufe (Wittchen/Jacobi 2005).

(2) Es zeigt sich eine hohe Komorbidität bei der Diagnose psychischer Erkrankungen (Hoyer 2006). Damit verbunden ist auch eine seltene Früherkennung und eine seltene adäquate Behandlung.

(3) Fast $80 \%$ der jungen Beschäftigten gehen zur Arbeit, auch wenn sie krank sind (DGB-Bundesvorstand 2010). Das entspricht der höchsten Präsentismusrate aller Altersgruppen.

(4) $45 \%$ junger Beschäftigter mit „schlechter Arbeit" berichten von regelmäßigen Schlafstörungen (DGB-Bundesvorstand 2010).

(5) Die meisten AU-Tage aufgrund psychischer Störungen weisen Erwerbstätige im mittleren Alter auf, wobei die Jüngeren deutlich aufholen (Jakobi 2009). Die Erkrankungsdauer bei psychischen Erkrankungen steigt durchschnittlich um $25 \%$ zwischen den 20- bis 24-Jährigen und den 25- bis 29-Jährigen. Als Ursachenbündel für die psychischen Belastungen werden Arbeitstempo, Termindruck, Arbeitsprozesse, Kommunikation und Zukunftsangst angeführt (Barmer Ersatzkasse 2009).

(6) Signifikante Auswirkungen psychischer Belastungen in der Adoleszenz auf das weitere Leben sind eine negative Sicht auf sich selbst, die Umwelt und die Zukunft, verbunden mit unreifen Konfliktbewältigungsstrategien, Lernhemmungen, übersteigerter Form der Sinnlosigkeit, des Versagens und Minderwertigkeitsgefühle (vgl. auch Egle 2005). Daher ist die Stärkung des Kohärenzgefühls bei jungen Beschäftigten bedeutend (Wydler et al. 2006).

Hieraus wird deutlich, welche Relevanz der beruflichen Stabilität - insbesondere bei jungen Erwerbstätigen - zukommt. Gerade mit den Erfahrungen, die Jugendliche und junge Erwachsene mit und auf dem Arbeitsmarkt sammeln - also Arbeitslosigkeit, Langzeitpraktika und atypische Beschäftigungsverhältnisse wie Leiharbeit -, sind weitreichende Folgen für die (Weiter-)Entwicklung des (Berufs-)Lebens verbunden. Die mehrheitlich jungen Arbeitskräfte in atypischer Beschäftigung erhalten weniger Entgelt als Beschäftigte 
im Normalarbeitsverhältnis, werden in den Betrieben strukturell ausgegrenzt und sind insgesamt vermehrten körperlichen, aber vor allem auch komplexen psychischen Belastungen ausgesetzt, wie die Ursache-Wirkungs-Kette deutlich macht. Am Beispiel Leiharbeit wurde gezeigt, dass die Arbeitssituation für die Betreffenden eine signifikant höhere psychische Belastungskonfiguration darstellt als im Normalarbeitsverhältnis.

Des Weiteren werden die mehrheitlich jüngeren Leiharbeitskräfte daran gehindert, die für das zukünftige Arbeitsleben wichtigen Gesundheitsressourcen aufzubauen. Diese sind zu verstehen als salutogene Faktoren, die es Personen erlauben, späteren Stress- und vor allem psychischen Belastungssituationen angemessen zu begegnen. Salutogene Faktoren helfen, Stress zu verarbeiten, Resilienz aufzubauen und die Person damit widerstandsfähiger gegen gesundheitliche Belastungen und Risikofaktoren zu machen (Bauer/Jenny 2007). Hierzu ist es wichtig, für die jungen Arbeitnehmer und Arbeitnehmerinnen betriebliche, personale und soziale Gesundheitsressourcen wie Handlungsspielraum, soziale Unterstützung, Partizipation, Lern- und Entwicklungsmöglichkeiten bereitzustellen und zu fördern (Langhoff et al. 2010). Ein weiterer wesentlicher Aspekt, um die Belastungssituation der (jungen) Arbeitnehmer in atypischen Beschäftigungsverhältnissen $\mathrm{zu}$ verbessern, ist die Realisierung des schon seit Langem geforderten gleichen Lohns für gleiche Arbeit sowie die Festlegung gesetzlicher Mindestlöhne.
Arbeitgeber sind daher aufgefordert, soziale Verantwortung für den langfristigen Erhalt der Arbeitsfähigkeit der jungen Erwachsenen zu übernehmen. Hierunter fällt z.B. auch das Fördern der Übernahme von Auszubildenden in Normalarbeitsverhältnisse. Aber auch die besonderen Belastungszusammenhänge für die Leiharbeitnehmer sind in der Zusammenarbeit mit dem Zeitarbeitsunternehmen in ihrer Wirkung zu minimieren. Echte Perspektiven durch Übernahmechancen können hier einen entscheidenden Beitrag zur Entlastung und zum Erhalt psychischer Gesundheit leisten.

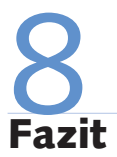

Jugendliche und junge Erwachsene sehen sich beim Eintritt ins Berufsleben mit zahlreichen Schwierigkeiten konfrontiert. Darunter fallen u. a. die vergebliche Suche nach einem passenden Ausbildungsplatz, die Zunahme (ausschließlich) prekärer Beschäftigungsangebote wie Dauerpraktika oder Leiharbeit sowie drohende Arbeitslosigkeit. Diese Entwicklung bedeutet für die Betroffenen nicht nur große finanzielle, sondern ebenso komplexe psychische Belastungssituationen.

Es wurde gezeigt, dass die Mehrheit der psychischen Erkrankungen sich bereits im Jugendalter manifestiert. Es wurde auch erläutert, dass insbesondere mit prekären Beschäftigungsverhältnissen wie Leiharbeit, der überproportional viele junge
Erwerbstätige nachgehen (müssen), besondere psychische Belastungen verbunden sind. Die Nicht-Zugänglichkeit zum Arbeitsmarkt hat für die betroffenen Personen also weitreichende Konsequenzen. Vor dem Hintergrund des demografischen Wandels ist es nun zwingend erforderlich, die physische und psychische Gesundheit der jungen Generation zu fördern statt dauerhaft zu belasten. Es geht auch darum, diese Gruppe damit langfristig arbeitsfähig zu erhalten. Davon profitieren die Jugendlichen selbst und schließlich auch die Gesellschaft, wenn die jungen Erwachsenen nämlich in ihrer Rolle als künftige Rentenbeitragszahler auch maßgeblich für den Erhalt der Sozialsysteme sorgen.

Wissenschaftliche Konzepte, Ideen und das Gestaltungswissen zur Bereitstellung und Implementierung der Gesundheitsressourcen bei der Arbeit liegen bereits vor (z. B. Gratifikationskrisenmodell, Siegrist 1996; Anforderungs-Kontroll-Modell, Karasek/Theorell 1990). Die Erkenntnisse werden bisher aber nur unzureichend umgesetzt. Wichtig in diesem Zusammenhang ist z. B. die zwingende Umsetzung der Gefährdungsbeurteilung unter Einbezug psychischer Belastungen (wie im Arbeitsschutzgesetz gefordert). Darüber hinaus wären politische und strukturelle Regelungen zur Vermeidung prekärer Arbeitsbedingungen notwendig (z. B. Praktika als Lernverhältnis und nicht als Ersatz für reguläre Arbeit), um insgesamt pathogene Entwicklungen der Gesundheit junger Beschäftigter einzudämmen und die Arbeitsfähigkeit der Betreffenden langfristig zu fördern. 
Bardasi, E./Francesconi, M. (2004): The impact of atypical employment on individual wellbeing. Evidence from a panel of British workers, in: Social Science and Medicine 9, S. 1671-1688

Barmer Ersatzkasse (2009): Gesundheitsreport 2009, Wuppertal Bauer, G/Jenny, G. (2007): Gesundheit in Wirtschaft und Gesellschaft, in: Moser, K. (Hrsg.): Wirtschaftspsychologie, Heidelberg, S. 221-243 Bundesanstalt für Arbeitsschutz und Arbeitsmedizin (BAuA)/Bundesministerium für Arbeit und Soziales (BMAS) (2008): Sicherheit und Gesundheit bei der Arbeit 2006. Unfallverhütungsbericht Arbeit, Dortmund, Berlin, Dresden

Bundesagentur für Arbeit (BA) (2009): Der Arbeits- und Ausbildungsmarkt in Deutschland, Monatsbericht 09, Nürnberg

Bundesinstitut für Berufsbildung (BIBB) (2008): BIBB/BAuA-Erwerbstätigenbefragung 2006 - Ergebnisse online: http://www.bibb.de/de/ 26901.htm

Bundesministerium für Bildung und Forschung (BMBF) (2009): Berufsbildungsbericht 2009, Bonn, Berlin

Deutsche Shell (Hrsg.)(2002): Jugend 2002 - Zwischen pragmatischem Idealismus und robustem Materialismus, Frankfurt a.M.

Deutscher Gewerkschaftsbund (DGB) Bundesvorstand (Hrsg.) (2010): Arbeitsqualität aus der Sicht von jungen Beschäftigten. Sonderauswertung des DGB-Index Gute Arbeit 2009, Berlin

Egle, U.T. (2005): Frühe Stresserfahrungen - Langzeitfolgen für die Gesundheit, Vortrag auf dem 1. Nationalen Präventionskongress, Dresden

Fischer, G. et al. (2008): Langfristig handeln, Mangel vermeiden: Betriebliche Strategien zur Deckung des Fachkräftebedarfs, IAB Forschungsbericht 03, Nürnberg

Fuchs, T. (2008): Was ist gute Arbeit? Anforderungen an den Berufseinstieg aus Sicht der jungen Generation. Ergebnisse einer repräsentativen Befragung von Berufseinsteigern im Alter zwischen 18 und 34 Jahren mit abgeschlossener Berufsausbildung; im Auftrag der Bundesanstalt für Arbeitsschutz und Arbeitsmedizin (BAuA) und des Bundesministeriums für Arbeit und Soziales (BMAS), Stadtbergen

Generation P (2007): Für faire Praktika und einen besseren Berufseinstieg von jungen Europäern, Petition an das Europaparlament, online: www.petition-p.org

Grau, A. (2010): Befristete Beschäftigung: Jeder elfte Vertrag hat ein Verfallsdatum. Statistisches Bundesamt. (STATmagazin), online: http:// tinyurl.com/2da9pfq aktualisiert am 16.03.2010.

Hans-Böckler-Stiftung (HBS)/Deutscher Gewerkschaftsbund (DGB) (Hrsg.) (2009): Konferenzdokumentation: Interessen und Interessenvertretung von jungen Prekären, 1. April 2009, Berlin

Hoyer, J. (2006): Bestandsaufnahme zur psychischen Gesundheit, Vortrag im Expertengespräch mit der SRH Dresden, Dresden

Institut für Arbeitsmarkt und Berufsforschung (IAB) (2007): Betriebspraktika - Auf Umwegen zum Ziel, IAB-Kurzbericht 7, Nürnberg
Jacobi, F. (2009): Nehmen psychische Störungen zu? Report Psychologie 1, S. 16-28

Karasek, R./Theorell, T. (1990): Healthy Work. Stress, Productivity and the Reconstruction of the Working life, New York

Kock, K. (2008): Auf Umwegen in den Beruf. Destandardisierte und prekäre Beschäftigung von Jugendlichen an der zweiten Schwelle - eine Auswertung empirischer Befunde im Auftrag der Hans-Böckler-Stiftung, Sozialforschungsstelle Dortmund, Beiträge aus der Forschung 162,

Dortmund

Langhoff, T./Satzer, R./Ertel, M. (2010): Erfahrungen zur Umsetzung der Gefährdungsbeurteilung bei psychischen Belastungen, Forschungsprojekt im Auftrag der BAuA, in: Arbeit 2010, Veröffentlichung in Vorbereitung

Mörchen, M. (2006) : Praktikum als prekäre Beschäftigung - Ausbeutung oder Chance zur Integration?, Sozialforschungsstelle Dortmund, Beiträge aus der Forschung 149, Dortmund

Prager, J./Wieland, C. (2005): Jugend und Beruf. Repräsentativumfrage zur Selbstwahrnehmung der Jugend in Deutschland, im Auftrag der Bertelsmann Stiftung, Gütersloh

Rump, J./Eilers, S. (2009): Ökonomische Effekte des Age Managements, Sternenfels

Sczesny, C./Schmidt, S./Schulte, H./Dross, P. (2008): Zeitarbeit in Nordrhein-Westfalen. Strukturen, Einsatzstrategien, Entgelte, Dortmund

Siegrist, J. (1996): Soziale Krisen und Gesundheit: Eine Theorie der Gesundheitsförderung am Beispiel von Herz-Kreislauf-Risiken im Erwerbsleben, Göttingen

Siegrist, J./Dragano, N. (2008): Psychosoziale Belastungen und Erkrankungsrisiken im Erwerbsleben, in: Bundesgesundheitsblatt - Gesundheitsforschung - Gesundheitsschutz 3, S. 305-312, online: http://dx.doi.org/10.1007/s00103-008-0461-5.

Stangl-Taller, W. (2009): (Arbeitsblätter) Arbeitslosigkeit bei Jugendlichen, online: http://arbeitsblaetter.stangl-taller.at/ (zuletzt am 28.09.2009)

Statistisches Bundesamt (2008): Destatis, Atypische Beschäftigung auf dem deutschen Arbeitsmarkt, Pressegespräch 09.09., Frankfurt am Main

Tivig, T./Hetze, P. (Hrsg.) (2007): Deutschland im demografischen Wandel, Rostocker Zentrum zur Erforschung des demografischen Wandels, Rostock

Vester, F. (2001): Die Kunst, vernetzt zu denken, München Wesfreid, M. (2006): Stages - La grande loterie, L'Express, Paris Wittchen, H.-U./Jacobi, F. (2005): Psychische Störungen in Deutschland und der EU, Zusammenfassung, online: http://www.tu-dresden. de/presse/psyche.pdf, Dresden

Wydler, H./Kolip, P./Abel, T. (Hrsg.) (2006): Salutogenese und Kohärenzgefühl: Grundlagen, Empirie und Praxis eines gesundheitlichen Konzepts 